\title{
A importância da higienização das mãos na saúde do pré adolescente: um relato de experiência
}

The importance of hand hygiene in pre-adolescent health: an experience report

La importancia de la higiene de manos en la salud preadolescente: un informe de experiencia

Danielle Freire Goncalves

ORCID: https://orcid.org/0000-0002-2469-1876 Universidade do Estado do Pará, Brasil

E-mail: Adaniellefreire@gmail.com

Khilver Doanne Sousa Soares

ORCID: https://orcid.org/0000-0002-0237-2890

Faculdade de Medicina de Marabá, Brasil E-mail: Khilver@gmail.com

Gabriela Cordeiro Silva

ORCID: https://orcid.org/0000-0002-0671-5694

Faculdade de Medicina de Marabá, Brasil

E-mail: Gab_cordeiro@outlook.com

Iasmim Ianne Sousa Tavares

ORCID: https://orcid.org/0000-0003-2350-874x

Faculdade de Ensino Superior da Amazonia Reunida, Brasil

E-mail: Iasmim.tavares14@gmail.com

Yuri Davi Vidal de Azevedo

ORCID: https://orcid.org/0000-0001-9838-3609

Universidade do Estado do Pará, Brasil

E-mail: Yuridavi02@gmail.com

Maria Lohane Castilho de Almeida

ORCID: https://orcid.org/0000-0002-2821-9739

Faculdade de Medicina de Marabá, Brasil

E-mail: Lohanyalmeida10@ hotmail.com

Milena Brandão Rios

ORCID: https://orcid.org/0000-0002-1110-1419

Faculdade de Medicina de Marabá, Brasil

E-mail: Milenabrandao74@gmail.com

Luciana Ferreira Gouveia Silva

ORCID: https://orcid.org/0000-0002-5701-7853

Faculdade de Medicina de Marabá, Brasil

E-mail: Lu20gouveia@hotmail.com

Marianne da Guia Fernandes da Costa Oliveira

ORCID: https://orcid.org/0000-0002-4959-9803

Faculdade de Medicina de Marabá, Brasil E-mail: Mariannedaguia_@gmail.com

Pedro Paulo Moraes da Câmara

ORCID: https://orcid.org/0000-0003-1338-8692 Universidade Federal do Pará, Brasil E-mail: Pedrocamaranutri@gmail.com

João Vitor de Menezes santos

ORCID: https://orcid.org/0000-0002-4371-0083

Faculdade de Medicina de Marabá, Brasil, Brasil

E-mail: Jvmsmed@gmail.com

João Paulo Dias Nogueira

ORCID: https://orcid.org/0000-0002-7904-4510

Faculdade de Medicina de Marabá, Brasil

E-mail: Jpnogueira02@gmail.com

Jose Wneyldson da Silveira

ORCID: https://orcid.org/0000-0002-7910-6339

Faculdade de Medicina de Marabá, Brasil

E-mail: Wneyldsonsilveira@gmail.com

Talyta Borges Cordeiro

ORCID: https://orcid.org/0000-0002-5964-3284

Faculdade de Medicina de Marabá, Brasil

E-mail: Talyta_borges20@hotmail.com

Michelle Quaresma Cardoso

ORCID: https://orcid.org/0000-0002-8621-8670

Universidade do Estado do Pará, Brasil

E-mail: Michelle.card91@yahoo.com.br 


\title{
Resumo
}

A fase da pré adolescência é marcada por várias mudanças psicossociais, por isso faz-se necessário sensibilizarmos positivamente as práticas cotidianas desse grupo. A higienização das mãos é uma prática indispensável na saúde da população em geral, sendo assim, é indubitavelmente necessário a introdução de tal rotina na vida do pré adolescente. O objetivo deste trabalhar é relatar a experiência vivida por acadêmicos do curso de enfermagem em uma ação de educação em saúde realizada em uma escola pública, com a temática de formas alternativas de higienização das mãos se baseando na realidade vivida por tal grupo. A metodologia utilizada foi a teoria da problematização proposta por Berbel, além de uma explicação sobre o tema e um quiz com prêmios para aumentar o interesse dos jovens sobre o trabalho. Os resultados obtidos foram postos levando em consideração a mudança de hábito logo percebida pelos pesquisadores. Com isso, vê-se a importância de levarmos a educação em saúde para a comunidade e assim minimizar os problemas vividos pela população.

Palavras-chave: Higienização das mãos; Educação em saúde; Enfermagem.

\begin{abstract}
The pre-adolescent phase is marked by several psychosocial changes, so it is necessary to positively sensitize the daily practices of this group. Hand hygiene is an essential practice in the health of the general population, so it is undoubtedly necessary to introduce such a routine in the life of pre-adolescents. The objective of this work is to report the experience of nursing students in a health education action carried out in a public school, with the theme of alternative forms of hand hygiene based on the reality experienced by this group. The methodology used was the problematization theory proposed by Berbel, in addition to an explanation about the theme and a quiz with prizes to increase the interest of young people about work. The results obtained were considered taking into account the change in habit that was soon noticed by the researchers. With this, we see the importance of taking health education to the community and thus minimizing the problems experienced by the population.
\end{abstract}

Keywords: Hand disinfection; Health education; Nursing.

\section{Resumen}

La fase preadolescente está marcada por varios cambios psicosociales, por lo que es necesario sensibilizar positivamente las prácticas cotidianas de este grupo. La higiene de manos es una práctica fundamental en la salud de la población en general, por lo que sin duda es necesario introducir dicha rutina en la vida de los preadolescentes. El objetivo de este trabajo es reportar la experiencia de estudiantes de enfermería en una acción de educación en salud realizada en una escuela pública, con el tema de formas alternativas de higiene de manos a partir de la realidad vivida por este grupo. La metodología utilizada fue la teoría de la problematización propuesta por Berbel, además de una explicación sobre el tema y un cuestionario con premios para incrementar el interés de los jóvenes por el trabajo. Los resultados obtenidos se consideraron teniendo en cuenta el cambio de hábito que pronto notaron los investigadores. Con esto, vemos la importancia de llevar la educación en salud a la comunidad y así minimizar los problemas que vive la población.

Palabras clave: Desinfección de las manos; Educación en salud; Enfermería.

\section{Introdução}

A higienização das mãos faz-se necessária para a prevenção de doenças infectocontagiosas e/ou infectoparasitárias, assim, é notório a importância da criação de programas e projetos de intervenção, os quais orientem e conscientizem os préadolescentes sobre a total relevância da higienização das mãos de forma correta, para a manutenção de uma vida saudável. O trabalho tem o intuito de conscientizar os alunos da turma do $7^{\circ}$ do ensino fundamental II de uma escola do município de Belém sobre a importância de higienização das mãos, além disso, informar sobre o tema, explicando os benefícios da higienização das mãos e os malefícios da não ação, e por fim, promover a alto-reflexão em relação a higiene no âmbito escolar.

A fundadora da enfermagem moderna, Florence Nightingale, caracterizou a lavagens das mãos adequada como o principal meio de controle de doenças e assim revolucionou o fazer saúde, minimizando consideravelmente as infecções no ambiente hospitalar (Coelho, et. Al, 2011).

A adolescência/ pré-adolescência é uma fase cheias de transformações biológicas e comportamentais. Inerente ao desenvolvimento humano, a adolescência é uma fase do desenvolvimento humano, semi-patológica, cheios de conflitos 
"naturais" (Mercês, 2007). Nesse sentido, é importante que nessa fase complicada e duvidosa, sejam introduzidos nos préadolescente hábitos muito relevantes para o desenvolvimento sadio de suas vidas. Hábitos como higienização das mãos são de total importância para a manutenção da saúde desses adolescentes, o qual, segundo a OMS (organização mundial da saúde) evita a contaminação por agentes infecciosos e por diversas vezes, pode evitar que o indivíduo adquira doenças como hepatite A, gripe, diarreias, entre outras.

Segundo dados da OMS, a simples ação de lavar as mãos pode reduzir em aproximadamente $40 \%$ os casos de contaminação por bactérias, parasitas, fungos e vírus, os quais causam inúmeras doenças ao organismo. Além disso, uma das consequências da má higienização das é a possibilidade de contaminação por bactérias multirresistentes. Ainda segundo a OMS, atualmente, cerca de meio milhão de pessoas ao redor do mundo sofrem de infecções causadas por bactérias super-resistentes a antibióticos. No brasil, de acordo com pesquisas da Agência Nacional de vigilância sanitária (ANVISA, 2017), cerca de 25\% das infecções são causadas por micro-organismos multirresistentes, sendo imunes a antibióticos.

De posse dos dados, é notório a importância da criação de programas e projetos de intervenção, os quais orientem e conscientizem os pré-adolescentes sobre a total relevância da higienização das mãos de forma correta, para a manutenção de uma saúde e vida saudável. Nessa lógica, a escola como instituição parceira e educadora dos alunos, a qual corrobora com o desenvolvimento do cidadão, deve estar disposta, organizada e preparada para orientar esses adolescentes sobre como a simples higienização das mãos influencia e influenciará na homeostasia do corpo desses indivíduos, bem como a manutenção de sua saúde.

Sendo as mãos um veículo de patógenos, a falta de higienização delas podem causar infecções tanto leves como fatais, sendo a lavagens das mãos uma das barreiras mais importante para a quebra desse veículo (Gauer, 2017).

A Higienização das mãos é uma prática indispensável para a saúde do pré-adolescente e uma prática de baixo custo e fácil acesso, na falta de água potável e/ou sabão, pode ser utilizado medidas alternativas como o álcool em gel para a realização da manutenção da higiene pessoal (Pedrosa, 2015).

A Organização Mundial da Saúde (OMS) demostrou que a diarreia nas últimas décadas foi umas das principais causas de morte infantil, uma estatística que poderia ser reduzida com profilaxias de baixo custo e fácil acesso para as populações mais carentes que são as principais vítimas dessa condição (Batista, 2017).

Sendo a escola é o meio onde os pré-adolescente passam uma parte considerável da sua semana, onde se alimentem, brincam, convive com outras pessoas e estudam (Batista 2015). Assim, novos comportamentos positivos podem ser desenvolvidos se houver o incentivo externo.

A educação em saúde é indubitavelmente necessária para a melhoria de vida da população, pois trata-se uma medida de prevenção à patologias de todos os níveis (Piantino, 2018).

Para lidar com o público infanto-juvenil, o uso de práticas lúdicas e jogos é uma alternativa para atrair a atenção e o interesse do público e assim ter o impacto desejado nos hábitos de vida deles (Souza, 2018).

A ação tem como objetivo minimizar as infecções decorrentes da falta de higienização das mãos, além disso, buscar modos alternativos para sensibilizar os pre adolescente e contornar os obstáculos encontrando-se dentro do ambiente escolar, como a falta de água e sabão que é algo recorrente no cotidiano do público alvo.

\section{Metodologia}

A metodologia utilizada para a realização deste trabalho foi Arco de Maguerez, composto por cinco etapas a serem concluídas através da problematização da realidade. Para a coleta de dados, foram realizadas entrevistas, observações e aplicação pratica que ocorreram durante o intervalo de aula dos pré-adolescentes. Além disso, foi usado a metodologia da problematizacão 
de Berbel, onde é proposto uma observação baseada em problematizar a situação para se buscar já forma de minimizar os impactos do problema (Berberl, 1995).

Local: A escola consiste em um prédio com três andares. No térreo, funciona a parte administrativa; uma lanchonete, a cozinha e em frente há um espaço com duas grandes mesas com bancos acoplados e adjunto a cozinha fica um dos banheiros da instituição. Ademais, encontra-se uma rampa de acesso para cadeirantes e ao lado uma sala de informática. No segundo andar encontram-se as salas, dois banheiros próximos à escada e um laboratório multidisciplinar e o terceiro andar possui apenas salas de aula.

A prática ocorreu em duas salas de aula do sétimo ano localizadas no segundo andar do prédio, salas que suportam em média 25 alunos por turno, com dimensão de $10 \mathrm{~m}$ de largura por $15 \mathrm{~m}$ de comprimento, compostas com cadeiras e mesas de estudo, além de um quadro negro e ventiladores.

Equipamentos e Materiais: Para a coleta de dados, foram utilizados: caderno para anotações relevantes, canetas e celular para registros fotográficos. Para o projeto de ação foram utilizados: questionário para jogo estilo quiz, pequenos frascos de álcool em gel, dois celulares para cronometragem e registros fotográficos, brindes (pipoca e álcool em gel).

Participantes: Os estudantes e funcionários da instituição participaram do levantamento de dados na condição de 30 entrevistados. No dia da ação participaram 19 pré-adolescentes do $7^{\circ}$ ano, com faixa etária entre 11 a 16 anos.

Procedimentos: Em uma primeira visita a escola, o grupo fez uma observação geral do comportamento dos estudantes, ambiente e infraestrutura do local. Após primeiras análises deu-se foco nas entrevistas e nos possíveis problemas de saúde. Em seguida, iniciou-se o processo de teorização, levantamento de pontos chaves, hipótese de solução e, por fim foi feita a ação.

A ação consistiu em três etapas, sendo realização de uma palestra, quiz com perguntas sobre o tema abordado e a entrega dos brindes. A exposição foi realizada em sala de aula, no dia da aplicação do plano de ação, e visou o esclarecimento dos alunos sobre o quão importante é a higienização das mãos na saúde do pré-adolescente. Além de apresentar os malefícios da não higienização das mãos dos alunos no âmbito escolar e cotidiano, também foi exposto os benefícios de se higienizar as mesmas para a manutenção da saúde e bem-estar. Posterior a exposição foi demonstrado como higienizar as mãos com água, sabão e álcool em gel utilizando a técnica correta. Após isso, os alunos foram divididos em dois grupos para a realização do quiz com perguntas referentes a importância da higienização das mãos na saúde do pré-adolescente. Por fim, os universitários fizeram a entrega de brindes para os alunos participantes e professores da instituição.

\section{Resultados e Discussão}

Diante da problematização do alto índice de doenças infectocontagiosas transmitidas pelas mãos, foi aportado tal tema. A diarreia é uma doença causada pela falta de higienização, que é a segunda maior causa de morte entre criança e adolescentes (Unicef 2013). Desse modo, a ação educadora na escola da rede pública de ensino foi de suma importância para a sensibilização dos alunos sobre a estima dos hábitos de higiene pessoal, principalmente das mãos. Além do mais, após a realização da ação pode-se compreender a nítida alteração no comportamento dos pré-adolescentes.

No decorrer da atividade foi visto o interesse na explicação feita pelos acadêmicos, tanto pelos alunos como pela professora de ciências biológicas que elogiou o trabalho e comentou que ela iria dar continuidade no assunto durante as suas aulas. Pois no cotidiano do público alvo não é encontrado ações que visam ajudar a comunidade a reduzir problemas fáceis de serem solucionadas. 
Ademais, a realização do "quiz" foi de relevância para a fixação dos assuntos aportados, com um resultado de $90 \%$ de acertos, o qual trouxe satisfação para os acadêmicos de enfermagem que disponibilizaram tempo e recursos para estar devolvendo o conhecimento para a comunidade. Quando um grupo de acadêmicos tem uma prática com essas bases, é uma forma de fazer saúde, percebendo as necessidades da comunidade e se introduzindo naquela realidade (Almeida, 2018).

Com o fim da apresentação oral, houve a distribuição de brindes para os vencedores do quiz e álcool gel para o público em geral. Alem da distribuição de um item comestível para analisar se o público estudado utilização a metodologia de higienizar as mãos correta e pode ser analisado que mais da metade deles utilizaram o álcool corretamente, demonstrando a necessidade de atividades integradoras para minimizar as infeções causadas pela falta de higiene no ambiente escolar.

Assim, trabalhos como o Saúde nas Escolas trazem resultados positivos para a saúde pública, pois a profilaxia é a melhor maneira de reduzir as patologias juvenis (Sobrinho, et al, 2017).

\section{Conclusão}

A experiência vivenciada pelos discentes na ação educadora na escola contribuiu para a qualificação acadêmica, pois mostrou a realidade vivenciada pelas escolas públicas brasileiras, com falta de infraestrutura, como os banheiros da escola, os quais estavam sem condições mínimas de uso, e de políticas públicas que contribuem para a conscientização e intervenção na realidade descrita. Além disso, essa experiência ímpar que os discentes vivenciaram, adicionou conhecimento e aprendizado, colaborando também para o amadurecimento do senso crítico, e assim, integrando o aluno à comunidade. Destaca-se, no entanto, a necessidade de expansão e aprimoramento da ação proposta, abrangendo maior número de pré-adolescentes e favorecendo a integração de outros atores sociais.

Sendo a escola um meio que permeia uma grande parte da vida do pre adolescente, é indubitavelmente necessário, dentro desse ambiente, trazer ações de melhoria de vida (Silva, 2016).

Ressalta-se, que a atividade Integrada em Saúde, tem suma importância para os acadêmicos, pois leva-nos até a realidade, fazendo com que possamos aprender na prática, situações reais da área e contribuindo para a qualificação do futuro profissional. Além disso, as AISs possibilitam aos acadêmicos minimizar ou solucionar problemas existentes na comunidade no âmbito da saúde, levando-o desse modo, benefício para a sociedade e para o aprendizado dos precursores (Paes, 2017).

\section{Referências}

Agência Nacional de Vigilância Sanitária, ANVISA, (2017). Investigação E Controle De Bactérias Multirresistentes Gerência de investigação e Prevenção das Infecções e dos Eventos Adversos (Gipea) Gerência Geral de tecnologia em Serviço de Saúde (GGTES). https://www.anvisa.gov.br/servicosaude/controle/reniss/manual\%20_controle_bacterias.pdf

Almeidad, E. R., Moutinho, C. B., \& Leite, M. T. de S. (2016). Prática pedagógica de enfermeiros de Saúde da Família no desenvolvimento da Educação em Saúde*. Interface - Comunicação, Saúde, Educação, 20, 389-402. https://doi.org/10.1590/1807-57622015.0128

Batista A., \& Souza, D. (2015). Universidade Federal De Minas Gerais -Ufmg Curso De Especialização Em Formação Pedagógica Para Profissionais Da SaúdeCEFPEPS. https://repositorio.ufmg.br/bitstream/1843/BUBD-A3AG79/1/tcc_ufmg_2015_ead_pdf.pdf

Batista, M., Secretário, M., Schier Da, A., Conselho, C., Ana, C., Izoton De Sadovsky, D., Kátia, G., Brandt, M., Epifânio, M., Toporovski, S., Da, R., Carvalho, R., Gomes, Rodrigues, L., (2017). Guia, S., \& De Atualização, P. (n.d.). Diarreia aguda: diagnóstico e tratamento Departamento Científico de Gastroenterologia Presidente. https://www.sbp.com.br/fileadmin/user_upload/2017/03/Guia-Pratico-Diarreia-Aguda.pdf

Berbel, N. A. N. (1995). Metodologia da problematizacão: uma alternativa metosologica apropriada para o ensino superio. Semina: Ciências Sociais e Humanas http://www.uel.br/revistas/uel/index.php/seminasoc/article/view/9458/

Coelho, M. S., Silva Arruda, C., \& Faria Simões, S. M. (2011). Higiene de manos como estrategia fundamental en el control de infección hospitalaria: un estudio cuantitativo. Enfermería Global, 10(21). https://scielo.isciii.es/scielo.php?pid=S1695-61412011000100003\&script=sci_arttext\&tlng=pt

Gauer, D., Análise qualitativa e quantitativa da microbiota das mãos dos funcionários de um posto de saúde. (n.d.). Revista RBAC. http://www.rbac.org.br/artigos/analise-qualitativa-e-quantitativa-da-microbiota-das-maos-dos-funcionarios-de-um-posto-de-saude/

Mercês, A., \& Bock, B. (n.d.). http://www.scielo.br/pdf/pee/v11n1/v11n1a07. 
Research, Society and Development, v. 10, n. 11, e316101119567, 2021

(CC BY 4.0) | ISSN 2525-3409 | DOI: http://dx.doi.org/10.33448/rsd-v10i11.19567

Paes, C. C. D. C., (2017). A importância da abordagem da educação em saude: revisão de literatura. Revista Educação Em Saúde. http://200.133.3.238/index.php/revasf/article/viewArticle/944

Pedrosa, D. F. A. (2015, July 10). Saúde escolar no ensino fundamental: subsídios para a prevenção e controle da infecção. www.teses.usp.br website: https://www.teses.usp.br/teses/disponiveis/22/22132/tde-09102015-161452/en.php

Piantino, C. B., Vanin, A. C., Vieira, M., \& Souza, D. H. I. (2019). Propostas de ações educativas no ambiente escolar como prática de promoção da saúde. Ciência et Praxis, 11(21), 107-110. https://revista.uemg.br/index.php/praxys/article/view/3913/2260

Sobrinho, R. A. et al.(2017). Percepção dos profissionais da educação e saude sore o programa saude na escola. Revista Pesquisa Qualitativa, https://editora.sepq.org.br/rpq/article/view/77

Souza, A. P. de. (2018). Dilemas éticos na educação em saúde no programa Saúde na Escola: a vivência de profissionais de atenção básica do Centro de Saúde Escola Lapa. App.uff.br. https://app.uff.br/riuff/handle/1/9149

Silva, E. T. da, Rocha, A. B., \& Silva, F. A. da. (2016). Educação em saúde na escola: promovendo a saúde por meio do lúdico, da linguagem artística e da literatura infantil. JMPHC | Journal of Management \& Primary Health Care |ISSN 2179-6750, 7(1), 71-71. https://doi.org/10.14295/jmphc.v7i1.397

UNICEF. (2013). Unicef alerta: diarreia é segunda maior causa de morte entre crianças | ACMD. https://www.acmd.org.br/unicef-alerta-diarreia-e-segundamaior-causa-de-morte-entre-criancas/ 